\title{
Analysis of the Impact of Household Tourism Consumption Based on Multilevel Structural Equation Model
}

\author{
Ping Zhang 1 and Kaijun Cao $\mathbb{D}^{2}$ \\ ${ }^{1}$ College of Business Xinjiang University, Urumqi 830091, China \\ ${ }^{2}$ College of Tourism, Xinjiang University, Urumqi 830049, China \\ Correspondence should be addressed to Kaijun Cao; zaq@stu.xju.edu.cn \\ Received 1 January 2022; Accepted 25 January 2022; Published 18 February 2022 \\ Academic Editor: Hasan Ali Khattak \\ Copyright ( 2022 Ping Zhang and Kaijun Cao. This is an open access article distributed under the Creative Commons Attribution \\ License, which permits unrestricted use, distribution, and reproduction in any medium, provided the original work is \\ properly cited.

\begin{abstract}
With the development of the social economy and the improvement of people's living standards, tourism has become one of the important lifestyles of families. Family tourism is a new trend that will have no small impact on further developing tourism and boosting domestic demand. With tourism activities booming today, family tourism consumption is an area worthy of study. This study examines household tourism consumption using a hierarchical structural equation model to explore the factors influencing the latent variables of income structure, household, and regional characteristics of consumption structure. Further combining these potential variables and their influences, the formation and change of consumption structure were explored using hierarchical structural equation modeling. This is of academic value and theoretical significance in refining the application of the hierarchical structural equation model and enriching the use of latent variable factor analysis in consumption structure research. Finally, based on the characteristics of family tourism consumption and the problems of family tourism, some suggestions are made for the development of tourism.
\end{abstract}

\section{Introduction}

Families are the cells of society and one of the largest segments of the tourism industry [1]. Family tourism refers to whole families organizing trips together [2]. As a new trend, family tourism consumption will have a great impact on further developing tourism and boosting domestic demand [3]. In the past, the tourism products launched in China were generally mass oriented, providing services mainly for ordinary tourists, and the main mode of travel was in the form of casual groups, which was not suitable for the requirements of family tourism development, and the focus of research on family tourism was concentrated on adults and neglected the analysis of the influence of children on family tourism decisions [4]. The analysis of family tourism consumption issues not only enriches the study of tourism consumer behavior but also helps tourism industry operators to gain a comprehensive understanding of family tourism consumption and preferences and to develop products for tourism [5]. It also helps tourism operators to gain a comprehensive understanding of the consumption needs and preferences of family tourism consumers and provides useful guidance for the development of tourism products and marketing strategies. Figure 1 shows the overall situation of domestic tourism in the past ten years.

The core family is made up of a couple and their unmarried children. There are two main types: the complete nuclear family and the single-parent family [6]. The former refers to a family consisting of a couple and their unmarried children, while the latter refers to a nuclear family with only a mother or a father. In today's society, nuclear families are generally couples who have work and have only one or two children and are financially well off. The size of the family is inversely proportional to the amount of time the couple spends outdoors, so the nuclear family has more time and money to travel. Children play a more important role in the travel decisions of these families. Families, therefore, place particular importance on the educational value of travel for 


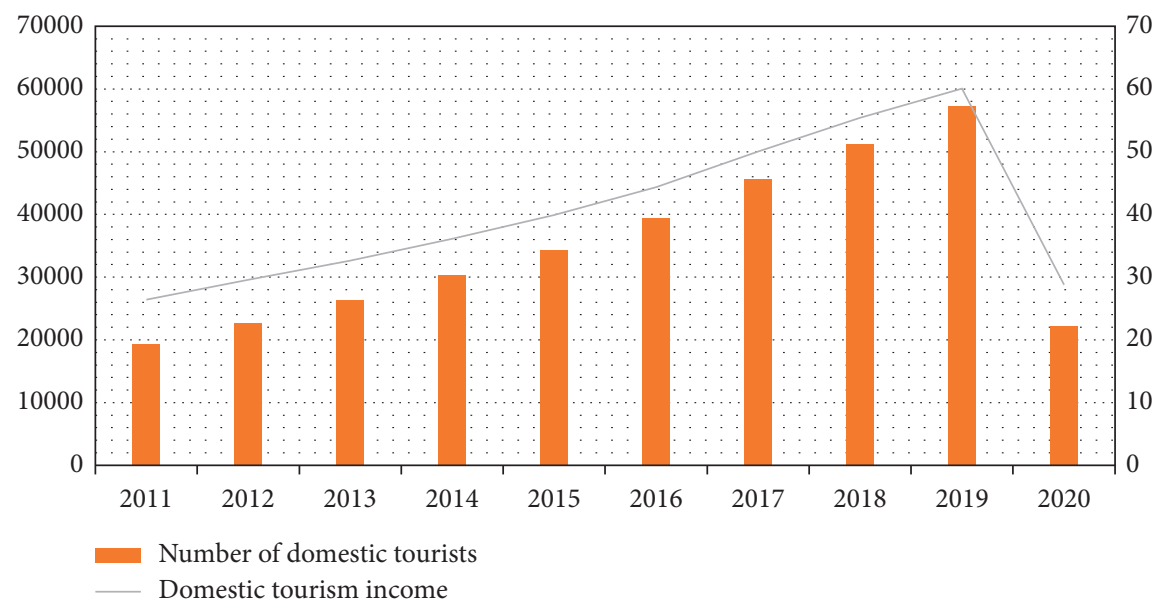

FIGURE 1: The overall situation of domestic tourism in the past ten years.

their children, and the timing of travel is influenced by their children's holidays, so the type of travel preferred by these families is more likely to be educational.

Income structure, as the core factor affecting the structure of household tourism consumption, is an important focus point for promoting the upgrading of tourism consumption structure [7]. One of the important reasons for the shift in the structure of household consumption toward development and enjoyment consumption is the increase in the level of household income and the increase in the sources of income of residents. People will use their wage income to spend on daily life and use the rewarding property income gains to travel or improve their quality of life. Changes in income structure, therefore, have a direct impact on the structure of household spending on tourism. This is why it is important to analyze the impact of different income types and other household factors on tourism consumption. The distribution of per capita monthly income of Chinese households is shown in Figure 2.

In addition, although China's tourism consumption market is improving, the regional disparity in tourism consumption is gradually increasing [8]. The economic differences between the eastern, central, and western regions have brought about a corresponding structural difference in household travel expenditure. The eastern region has the highest level, but the largest intraregional disparity, while the western region has the lowest level, but the smallest intraregional disparity. Tourism consumption is in urgent need of expansion and quality improvement within the internal structure. There is an urgent need to upgrade the structure of household tourism consumption, gradually achieving a shift from an increase in quantity to an upgrade in structure.

This study uses a multilevel structural equation model to explore the various influencing factors of the latent variables of household tourism consumption structure at three levels. The research in this study has implications for the promotion of the upgrading of the tourism consumption structure of residents in each region and the formulation of corresponding tourism consumption policies. In addition, it has some academic value and theoretical significance in

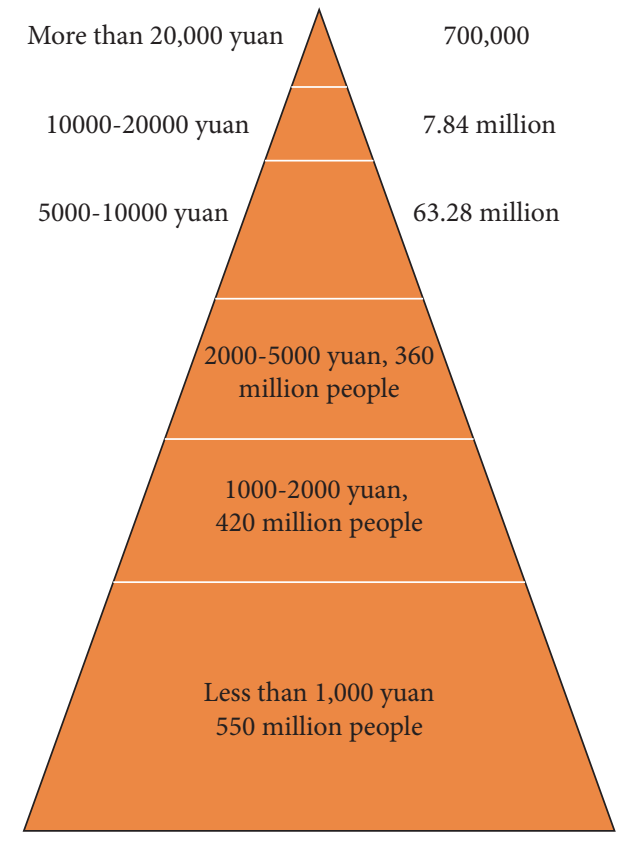

FIgURE 2: The distribution of per capita monthly income of Chinese households.

improving the model application of the hierarchical structural equation and enriching the use of latent variable factor analysis in consumption structure research.

\section{Related Work}

\subsection{Theory of Consumption}

2.1.1. Absolute Income Hypothesis. Keynes argued that consumption is a function of income, i.e., that consumption is primarily determined by income, while other factors affecting consumption have both positive and negative effects and can, therefore, be seen as having an offsetting influence $[9,10]$. This is the theoretical basis for this study's use of income as the main factor influencing the structure of 
household tourism consumption. Keynes classified consumption as spontaneous and induced, and the absolute income consumption function is as follows:

$$
C=\bar{C}+m p c * Y,
$$

where $C$ is people's total consumption, $\bar{C}$ refers to people's spontaneous consumption, i.e., consumption that meets people's basic needs, $m p c * Y$ refers to people's induced consumption, i.e., consumption that people undertake after meeting their basic needs, and mpc denotes people's marginal propensity to consume, with $0<m p c<1$.

2.1.2. Relative Income Hypothesis. Consumption is not only determined by the consumer's current income, but it is also influenced by the level of income compared to that of the past and those around them. According to the relative income hypothesis, the theory actually divides the income structure into current income and past income to examine the relationship between these two types of income on consumption $[11,12]$. So, when people determine the type of consumption, they are influenced by a combination of current and past income and the income of those around them. Therefore, in a region where the income gap or the urban-rural gap is too wide, due to the demonstration effect, low-income earners will follow the example of high-income earners in their pursuit of a high standard of living, and there will be consumption choices that increase consumption expenditure to follow suit even if their income has not increased, which may be reflected at the enjoyment consumption level is reflected.

2.1.3. Life-Cycle Hypothesis. The life-cycle hypothesis assumes that a person's consumption is determined by their income earned over a lifetime. People earn income through labor when they are young but do not spend all of it on consumption and income from labor when they are young but do not spend all of it on consumption; instead, they save some of it for property income, and when they are old and when they lose their source of income, they consume the income they have earned through savings in order to achieve an optimal allocation of consumption over the life cycle. The optimal allocation of consumption over the life cycle and the consumption function of the life-cycle hypothesis are as follows:

$$
C=a * W+c * Y,
$$

where $W$ is the wealth gain, $Y$ is the wage income, and $a$ and $c$ are respective for marginal propensity to consume.

It follows from the life-cycle hypothesis that the theory actually divides the income structure into property income and labor income. Since $a$ and $c$ are equal only in special cases, i.e., different sources of income generate different marginal propensities to consume, this provides a valuable theoretical basis for this chapter when examining the relationship between consumption structure and income structure.
2.1.4. Persistent Income Hypothesis. Friedman divides income from different sources into stable income and temporary income $[13,14]$. Stable income is defined as income that people can stable income that people can earn over time. Temporary income is income that is occasionally received and does not last. This theory suggests that people choose their consumption type not on the basis of temporary income but on the basis of stable income. It is only when temporary income rises to stable income that people adjust their consumption. The model for the persistent income hypothesis is as follows:

$$
C=c * Y_{P}=c * \theta Y_{t}+c *(1-\theta) Y_{t-1},
$$

where $c$ is the marginal propensity to consume, $Y_{P}$ is the stable income, $Y_{t}$ is the current period income, $Y_{t-1}$ is the previous period income, $\theta$ indicates the extent to which income changes affect stable income, and $c * \theta$ and $c *(1-$ $\theta)$ are the two-period average consumption propensity to consume. At this point, the income structure is divided into the income of the previous period and the income of the current period. According to the persistent income hypothesis, changes in persistent income cause changes in consumer spending. The persistent income hypothesis is concerned with studying the impact of income on consumption with a greater focus on the type of income, where a stable increase in income over time is conducive to increased consumption by residents.

2.2. Theoretical Model Construction. The Dixit-Stiglitz (D-S) model [15] and the Cobb-Douglas (C-D) production function model [16] are then used to construct a model of household tourism consumption with income structure factors, household, and regional factors and to investigate the relationship between income structure factors, household and regional factors, and residents' household tourism consumption. On the demand side of tourism, according to the Dixit-Stiglitz (D-S) model, it is assumed that the utility function obeys the CES utility function and that households' preferences for tourism products are homogeneous. At this point, the demand function for tourism products for nuclear households is as follows:

$$
U=\left[\sum_{j} n_{j} X_{j}^{\delta-1 / \delta}\right]^{\delta-1 / \delta}, \quad \delta>1,
$$

where $U$ denotes the utility of tourism consumption by regional households, the type of tourism product available in the $n$ market, and $X$ denotes the number of trips consumed by local households. $\delta$ denotes the constant tourism elasticity of substitution, and it is greater than 1. According to the principle of maximizing consumer utility, the optimal number of times a family travel consumer spends $X_{j}$ is obtained as follows:

$$
X_{j}=p_{j}^{-\delta}\left(\sum_{j=1}^{31} n_{j} p_{j}^{1-\delta}\right)^{1 / 1-\delta} C_{j} .
$$

At this point, let $G_{j}=\left(\sum_{j=1}^{\mathrm{N}} n_{j} p_{j}^{1-\delta}\right)^{1 / 1-\delta}$ and reduces to 


$$
X_{j}=p_{j}^{-\delta} G_{j}^{\delta-1} C_{j},
$$

where $p$ is the final amount paid by households for tourism consumption, and $G$ is the regional price index for consumer tourism. Then $C$ is as follows:

$$
C_{j}=X_{j} p_{j}^{\delta} G_{j}^{1-\delta} \text {. }
$$

According to the Cobb-Douglas (C-D) function model, it is assumed that there are only two-factor inputs to tourism supply, namely, labor $L$ and capital $K$, and that the type of tourism $A$ varies from region to region. The short-term tourism product supply function is as follows:

$$
F_{j}=A_{j} F\left(\bar{K}_{j}, L_{j}\right)=A_{j} L_{j}^{\alpha} K_{j}^{\beta},
$$

where $L_{j}$ is the tourism-related factor, $\alpha$ is the labor elasticity coefficient, $\overline{K_{j}}$ is the capital factor that is fixed in the short run, $\beta$ is the elasticity of capital output, and $0<\alpha, \beta<1$, $\alpha+\beta=1$. In this study, the specific function of tourism services $A$ is set in the context of the research on the impact of household and regional factors on residential household consumption as follows:

$$
A_{j}=\mathrm{Fam}_{j}^{\rho_{1}} \mathrm{GDP}_{j}^{\rho_{2}} e^{\rho_{3} \mathrm{MAR}_{j}},
$$

where $\mathrm{Fam}_{j}$ is a human capital factor measuring the labor force characteristics of households in the region. $\mathrm{GDP}_{j}$ is a physical capital factor measuring the level of economic development in the region. $\mathrm{MAR}_{j}$ is an external market factor measuring the level of marketization of the region. At this time, the short-term tourism product supply function is as follows:

$$
F_{j}=\operatorname{Fam}_{j}^{\rho_{1}} \mathrm{GDP}_{j}^{\rho_{2}} e^{\rho_{3} \mathrm{MAR}_{j}} L_{j}^{\alpha} K_{j}^{\beta} .
$$

Then, marginal cost $M C_{j}$ can be expressed as the product of the marginal output of labor $M P_{j}$ and the individual income of the worker $M_{i}$.

$$
\begin{aligned}
& M P_{j}=\frac{d F}{d L}=\alpha \operatorname{Fam}_{j}^{\rho_{1}} \operatorname{GDP}_{j}^{\rho_{2}} e^{\rho_{3} \mathrm{MAR}_{j}} L_{j}^{\alpha-1} K_{j}^{\beta}, \\
& M C_{j}=M_{i} * M P_{j}=M_{i} \alpha \operatorname{Fam}_{j}^{\rho_{1}} G D P_{j}^{\rho_{2}} e^{\rho_{3} \mathrm{MAR}_{j}} L_{j}^{\alpha-1} K_{j}^{\beta} .
\end{aligned}
$$

When supply and demand are in balance, $p_{j}=M C_{j}$, equation (12) is brought into $C_{j}$,

$$
C_{j}=M_{i}^{\delta} \alpha^{\delta}\left(\operatorname{Fam}_{j}^{\rho_{1}} G D P_{j}^{\rho_{2}} e^{\rho_{3} \mathrm{MAR}_{j}}\right)^{1+\delta} L_{j}^{\alpha+\alpha \delta-\delta} \bar{K}_{j}^{-\beta-\beta \delta} G_{j}^{1-\delta} .
$$
(14).

Taking the logarithm of equation (13) gives equation

$$
\begin{aligned}
\ln C_{j}= & \delta \ln M_{i}+\delta \ln \alpha+\rho_{1}(1+\delta) \ln \mathrm{Fam}_{j}+\rho_{2}(1+\delta) \ln \mathrm{GDP}_{j}+\rho_{3}(1+\delta) \mathrm{MAR}_{j} \\
& +(\alpha+\alpha \delta-\delta) \ln L_{j}-(\beta+\beta \delta) \ln \bar{K}_{j}+(1-\delta) G_{j} .
\end{aligned}
$$

First, the income $M_{i}$ is expanded to $M_{i}=\alpha i+\mathrm{dW}$ agei + eOperatei + fpropertyi + gtransferi. They, respectively, represent wage income, business income, property income, and transfer income. $d$, $e, f$, and $g$, respectively, represent the marginal propensity to consume the four types of income for different tourism consumption structures. Second, the factor of measuring family characteristics $\mathrm{Fam}_{j}$ is expanded to the size of the family population $\mathrm{Size}_{i}$, family urban and rural household registration $\mathrm{Urban}_{\mathrm{i}}$, years of education $E d u_{i}$, and years of work $\operatorname{Exp}_{i}$. Finally, the labor force $\ln L_{j}$ is rewritten as $\ln L_{j}=\ln \overline{\mathrm{MAN}}_{j}-\ln \left(1-\mathrm{OLD}_{j}^{\prime}-\mathrm{CHI}_{j}^{\prime}\right)$, where $\overline{\mathrm{MAN}}_{j}$ is the total population, $\mathrm{OLD}_{j}^{\prime}$ is the proportion of the elderly, and $\mathrm{CHI}_{j}^{\prime}$ is the proportion of children. At this time, the model is as follows:

$$
\begin{aligned}
\ln C_{j}= & \delta \ln \left(\alpha_{\mathrm{i}}+\text { dWage }_{i}+\text { eOperate }_{i}+\text { fProperty }_{i}+\text { gTransfer }_{i}\right)+\delta \ln \alpha \\
& +\rho_{1} 1+\left(\pi_{1} \text { Size }_{\mathrm{i}}+\pi_{2} \mathrm{Urban}_{i}+\pi_{3} \mathrm{Edu}_{i}+\pi_{4} \operatorname{Exp}_{i}\right)+\rho_{2}(1+\delta) \ln \mathrm{GDP}_{j}+\rho_{3}(1+\delta) \mathrm{MAR}_{j} \\
& +(\alpha+\alpha \delta-\delta) \ln \mathrm{MAN}_{j}+(\alpha+\alpha \delta-\delta) \ln \left(1-\mathrm{OLD}_{j}^{\prime}-C H I_{j}^{\prime}\right)-(\beta+\beta \delta) \ln \bar{K}_{j}+(1-\delta) G_{j} .
\end{aligned}
$$

Suppose $\quad C_{0}=\delta \ln \alpha-(\beta+\beta \delta) \ln \quad \bar{K}_{j}+(\alpha+\alpha \delta-\delta)$ $\ln \overline{\mathrm{MAN}}_{j}+(1-\delta) G_{m}$, then the above formula is simplified to: 


$$
\begin{aligned}
\ln C_{j}= & C_{0}+\delta \ln \left(\alpha_{\mathrm{i}}+d \text { Wage }_{i}+e \text { Operate }_{i}+\text { fProperty }_{i}+\text { gTransfer }_{i}\right) \\
& +\left[\rho_{1}(1+\delta) \ln \left(\pi_{1} \text { Size }_{i}+\pi_{2} \text { Urban }_{i}+\pi_{3} E d u_{i}+\pi_{4} \exp _{i}\right)\right] \\
& +\left[\rho_{2}(1+\delta) \ln G D P_{j}+\rho_{3}(1+\delta) M A R_{j}+(\alpha+\alpha \delta-\delta) \ln \left(1-\text { OLD }_{j}^{\prime}-C H I_{j}^{\prime}\right)\right] .
\end{aligned}
$$

The formula reveals the impact of income structure, household characteristics, and regional characteristics on tourism consumption, where $\ln C_{j}$ is the growth rate of tourism consumption and the first term on the right-hand side of the equation, $C_{0}$ is a constant parameter, the second term reflects the impact of income structure on it, the third term reflects the impact of household characteristics on it, and the fourth term reflects the impact of regional characteristics on it.

Based on the results derived from the model above, the following hypotheses are formulated and tested in the empirical analysis that follows:

(1) Income structure: income from wages (wage), income from business (operate), income from property (proper), and income from transfers (sfer) are related to household tourism consumption.

(2) Household characteristics: population size (size), urban and rural household size (urban), educational attainment (edu), and work experience (exp) are related to household tourism consumption.

(3) Regional characteristics: level of regional economic development (GDP), level of marketization (MAR), the proportion of the elderly population (OLD), and the share of the child population (CHI) are associated with household tourism consumption.

The impact of these three variables on tourism consumption is to be further determined in the later empirical section.

\section{Methods}

Based on the research idea, we will conduct a latent variable analysis, which requires the use of latent variable analysis to abstract out three levels of latent variables of household tourism consumption structure [17]. Second, this study involves a large number of research variables and needs to deal with the complex relationship of multiple variables to multiple variables and cannot simply use traditional regression. Based on the proposed theoretical model, household factors and regional factors that affect the structure of tourism consumption are also included in the analysis. However, as households from the same region tend to have greater similarity due to their enjoyment of common tourism resources, environment, etc., there is a high degree of aggregation in this nested structure of data, which does not meet the independence assumptions of the statistical model. Therefore, there is a need to define the structure of tourism consumption using appropriate measures and to study the relationships of the factors influencing it. The use of multilevel structural equation modeling (MSEM) [18] is required when it is necessary to incorporate latent variable models, to consider complex relationships between multiple variables, and to deal with nested structural data to analyze intergroup and intragroup differences. The theoretical foundations of the two most important models in hierarchical structural equation modeling, namely, hierarchical modeling and structural equation modeling, are presented in turn, followed by a summary of the theoretical foundations of hierarchical structural equation modeling.

3.1. Hierarchical Linear Model. The hierarchical linear model (HLM) is proposed to deal mainly with problems with nested data structures [19]. One of the most common applications of the hierarchical model one of the most common applications of HLM is the nesting of individuals within aggregates, e.g., households nested within different communities and individuals nested within different industries, in different industries. If, for example, households are used as variables at the individual level and regions are used as variables at the aggregate level, the hierarchical linear model takes the following form:

First level:

$$
Y_{i j}=\beta_{0 j}+\beta_{1 j} X_{i j}+\mu_{i j} .
$$

Second level:

$$
\begin{aligned}
& \beta_{0 j}=\alpha_{00}+\alpha_{01} Z_{j}+\varepsilon_{0 j} \\
& \beta_{1 j}=\alpha_{10}+\alpha_{11} Z_{j}+\varepsilon_{1 j} .
\end{aligned}
$$

Of these, $j=1,2, \ldots, j ; i=1,2, \ldots n_{j}, \operatorname{COV}\left(\mu_{i j}\right.$, $\left.\varsigma_{0 j}\right)=0, \operatorname{COV}\left(\mu_{i j}, \varsigma_{1 j}\right)=0$.

In the three equations above, $Y_{i j}$ is the household leveldependent variable and $X_{i j}$ is the independent variable, where the subscript $j$ represents different regions and $i$ represents households within a region. The household-level intercept and slope are $\beta_{0}$ and $\beta_{1}$, respectively. $\mu_{i j}$ is the random error term at the household level. $\alpha_{00}$ and $\alpha_{01}$ are the intercept and slope terms of the first equation at the second level, respectively. The meaning represented is the effect of the independent variable $Z_{j}$ in the second level on the firstlevel regression model intercept terms $\beta_{0 j}$ and $\beta_{1 j}$.

However, the multilevel linear model can only analyze the relationship between explicit variables and is very limited in many latent variable problems, so the following structural equation is the introduced model to solve this problem.

3.2. Structural Equation Modeling. Structural equation modeling (SEM) [20] is a statistical method similar to multivariate factor analysis, which has two main parts. One part constructs the relationship between the observed variables (i.e., indicators, usually explicit variables) and the 
latent variables (i.e., factors, usually concepts), and the other part constructs the interrelationships between the latent variables (i.e., correlations between individual factors).

$$
\text { Measurement model: }\left\{\begin{array}{l}
Y=\Lambda_{y} \eta+\varepsilon \\
X=\Lambda_{x} \xi+\delta
\end{array}\right.
$$

The measurement equation describes the relationship between the latent variable and the observable indicator of the explicit variable. Among them, $Y$ and $X$ are observed variables; $\eta$ and $\xi$ are latent variables; $\Lambda_{y}$ and $\Lambda_{x}$ are the factor loading between the latent variable and the observed variable, that is, the factor loading matrix; and $\varepsilon$ and $\delta$ are measurement errors.

$$
\text { Structural model: } \eta=\alpha+B \eta+\Gamma \xi+\zeta \text {. }
$$

The structural equation describes the relationship between latent variables. Among them, $\alpha$ is the intercept, $B$ and $\Gamma$ are the coefficient matrix between the latent variable and the latent variable, and $\zeta$ is the residual vector of the latent variable regression of the structural equation.

In summary, traditional regression analysis can only analyze the relationship between explicit variables, and the concept of latent variables, which cannot be directly observed, can only be roughly summarized using one or a few explicit variables. Structural equation modeling can, therefore, bridge the mapping process from "latent variables" to "observed explicit variables" by creating the concept of "latent variables," representing the structural relationship between latent variables and observed explicit variables. The current structural equation focuses on one level of analysis. However, this study uses multiple levels of data, both at the household and regional levels, which requires a break from the limitations of structural equation modeling in the use of such data to conduct multilevel analysis. Therefore, a hierarchical structural equation model is introduced next.

3.3. Multilevel Structural Equation Modeling. The multilevel structural equation modeling (MSEM) is a multilevel structural unified model that combines the strengths of both hierarchical linear models (HLMs) and structural equation models (SEMs) and is a multilevel latent variable model for the analysis of A generic framework for the analysis of multilevel latent variable models. The model is as follows:

$$
\text { Measurement model: } Y_{i j}=v_{j}+\lambda_{j} \eta_{i j}+\mathrm{K}_{j} X_{i j}+\varepsilon_{i j} \text {, }
$$

where $i$ is the individual indication, and $j$ is the cluster indication (representing the variation of this parameter with the cluster). The measurement equation can be viewed as a validated factor analysis expressing the observed variable $Y_{i j}$ as a function of the latent variable $\eta_{i j}$, the observed covariate $X_{i j}$, and the residual $\varepsilon_{i j}$.

At this point, the coefficients are allowed to vary between levels because of the need to reflect second-level random effects. The structural model is divided into two levels.

The within-group structural model:

$$
\eta_{i j}=\alpha_{j}+B_{j} \eta_{i j}+\Gamma_{j} X_{i j}+\zeta_{i j}
$$

The between-group structural model:

$$
\eta_{j}=\mu+\beta \eta_{j}+\gamma X_{j}++\zeta_{j}
$$

In the first-level intragroup structure model, the structural equation can be regarded as the expression of the functional relationship between the latent variable $\eta_{i j}$ and the exogenous observation covariate $X_{i j}$ and the residual $\zeta_{i j}$. In the second-level intergroup structure model, $X_{j}$ is the second-level s-dimensional covariate vector. Unlike $X_{i j}$, it is an s-dimensional vector of all group covariates stacked at the second level.

\section{Analysis of Household Tourism Consumption}

4.1. Data Sources and Descriptive Statistics. All data used in this study are from the China Household Tracking Survey (CFPS) microdata [21]. By combing through the sample data, data on total income, assets, travel consumption, and basic demographic information of each urban household (household) were obtained, and the sample excluded core variables with missing data or abnormal data. Descriptive statistics for the sample are shown in Table 1.

4.2. Family Tourism Consumption Structure. Since the implementation of the reform and opening up in 1978 [22], the structure of family tourism consumption in my country is undergoing drastic transformation and upgrading. The structure of tourism consumption in different periods has different performance characteristics. Figure 3 shows the changes in the structure of family tourism consumption.

From the above analysis, we can see that from 1992 to 1993, and from 2012 to 2013, the structure of family tourism consumption has undergone major changes. Therefore, using this as a node is used to analyze the changes in the average of the three stages. In the first stage, from 1978 to 1992, my country's family tourism consumption was dominated by subsistence consumption such as food and housing. The rest of the development and enjoyment consumption accounted for a relatively small proportion, while the consumption expenditure on transportation and other supplies and services accounted for a relatively small proportion, mainly for short-distance travel. In the second stage, during 1993-2012, reform and opening up were further deepened, and the tourism market changed. During this period, family tourism consumption was further upgraded, and the proportion of food expenditure decreased compared with the previous period. In the third stage, between 2013 and 2018, my country's economy is in a stage of rapid development, and tourism consumption is urbanizing and developing at a fast pace. During this period, the tourism consumption structure continues to upgrade. Although the growth rate of development and enjoyment tourism consumption is relatively slow, at this stage, the sum of expenditures on transportation, sightseeing, and shopping of Chinese residents has exceeded that of food and housing, 
TABLe 1: Descriptive statistics of the sample.

\begin{tabular}{|c|c|c|c|c|c|c|c|}
\hline \multirow{2}{*}{ Variable type } & \multirow{2}{*}{ Variable } & \multicolumn{2}{|c|}{ All samples } & \multicolumn{2}{|c|}{$\begin{array}{l}\text { Outbound travel } \\
\text { samples }\end{array}$} & \multicolumn{2}{|c|}{$\begin{array}{c}\text { Nonoutbound travel } \\
\text { samples }\end{array}$} \\
\hline & & Mean & $\begin{array}{l}\text { Standard } \\
\text { deviation }\end{array}$ & Mean & $\begin{array}{l}\text { Standard } \\
\text { deviation }\end{array}$ & Mean & Standard deviation \\
\hline Explained variable & $\begin{array}{l}\text { Per capita household travel } \\
\text { consumption }\end{array}$ & $0.10^{* *}$ & 0.31 & 0.25 & 0.44 & 0 & 0 \\
\hline \multirow{5}{*}{$\begin{array}{l}\text { Core explanatory } \\
\text { variables }\end{array}$} & Household assets per capita & $32.54^{* * *}$ & 78.05 & 64.46 & 98.4 & 28.07 & 76.69 \\
\hline & Per capita housing assets & $36.93^{* *}$ & 81.79 & 55.24 & 90.76 & 24.9 & 72.86 \\
\hline & Financial assets per capita & $3.51^{* *}$ & 11.61 & 5.9 & 14.87 & 1.93 & 8.47 \\
\hline & Operating assets per capita & $3.52^{*}$ & 12.76 & 0.06 & 0.76 & 0.03 & 0.2 \\
\hline & Durable goods assets per capita & $2.03^{* *}$ & 5.9 & 3.3 & 6.25 & 1.2 & 5.51 \\
\hline \multirow{9}{*}{ Control variable } & $\begin{array}{l}\text { Household disposable income per } \\
\text { capita }\end{array}$ & $4.83^{* * *}$ & 5.98 & 6.25 & 17.27 & 3.9 & 75.7 \\
\hline & Age of head of household & 48.65 & 15.17 & 47.99 & 15.57 & 48.91 & 16.78 \\
\hline & Gender of head of household & $0.51^{* *}$ & 0.5 & 0.47 & 0.5 & 0.54 & 0.49 \\
\hline & $\begin{array}{c}\text { Educational level of head of } \\
\text { household }\end{array}$ & $0.85^{*}$ & 0.68 & 0.8 & 0.69 & 0.87 & 0.76 \\
\hline & Head of household marriage & $0.82^{* *}$ & 0.39 & 0.8 & 0.4 & 0.85 & 0.48 \\
\hline & Number of family members & 3.39 & 1.66 & 3.43 & 1.74 & 3.33 & 1.78 \\
\hline & $\begin{array}{l}\text { Percentage of children among } \\
\text { family members }\end{array}$ & 0.16 & 0.38 & 0.17 & 0.46 & 0.16 & 0.45 \\
\hline & $\begin{array}{c}\text { Proportion of elderly in family } \\
\text { members }\end{array}$ & 0.05 & 0.06 & 0.07 & 0.09 & 0.04 & 0.07 \\
\hline & Family medical insurance & $0.47^{* *}$ & 0.4 & 0.61 & 1.29 & 0.43 & 1.54 \\
\hline
\end{tabular}

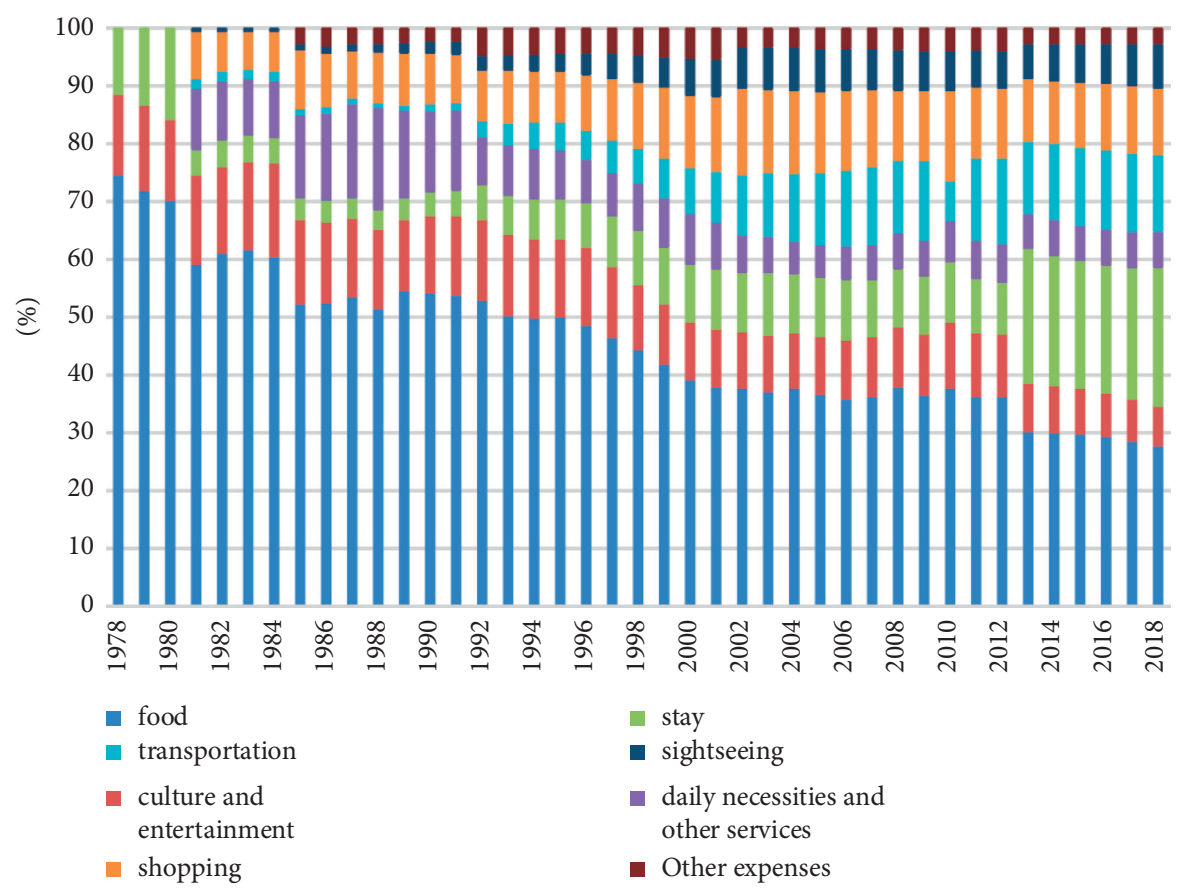

Figure 3: The changes in the structure of family tourism consumption.

which shows that people pay more attention to long-distance travel and enjoyment of life. The main reason is that changes in the lifestyle of family consumers have brought about changes in consumption concepts, and people are paying more and more attention to the quality of tourism.
4.3. Analysis by Rural and Urban Areas. China's special urban-rural dichotomy has gradually widened the gap between the consumption of urban and rural residents, and it is necessary to analyze the structure of household tourism consumption from both urban and rural perspectives. As 


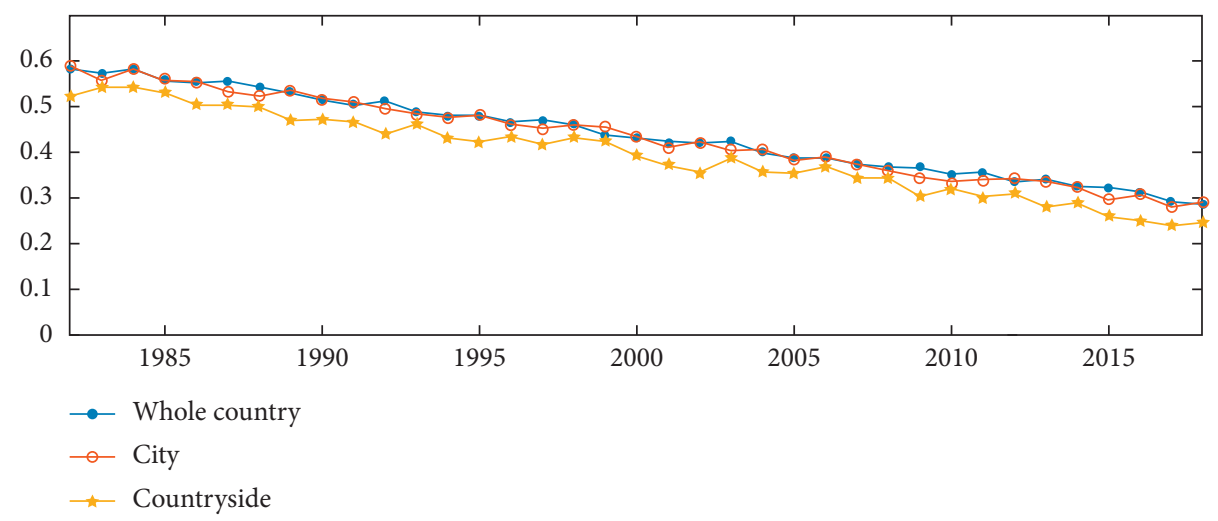

FIgURE 4: Changes in Engel's coefficient over the years.

TABLE 2: Income structure of urban residents.

\begin{tabular}{lcccc}
\hline Period & Wage & Operate & Property & Transfer \\
\hline $1978-1992$ & 76.13 & 1.49 & 1.03 & 21.75 \\
$1993-2012$ & 70.54 & 8.57 & 2.72 & 25.15 \\
$2013-2018$ & 60.80 & 11.25 & 10.09 & 17.86 \\
\hline
\end{tabular}

TABLE 3: Income structure of rural residents.

\begin{tabular}{lcccc}
\hline Period & Wage & Operate & Property & Transfer \\
\hline $1978-1992$ & 14.1 & 82.37 & - & 3.61 \\
$1993-2012$ & 28.14 & 63.29 & 2.13 & 6.44 \\
$2013-2018$ & 40.98 & 37.03 & 2.30 & 19.69 \\
\hline
\end{tabular}

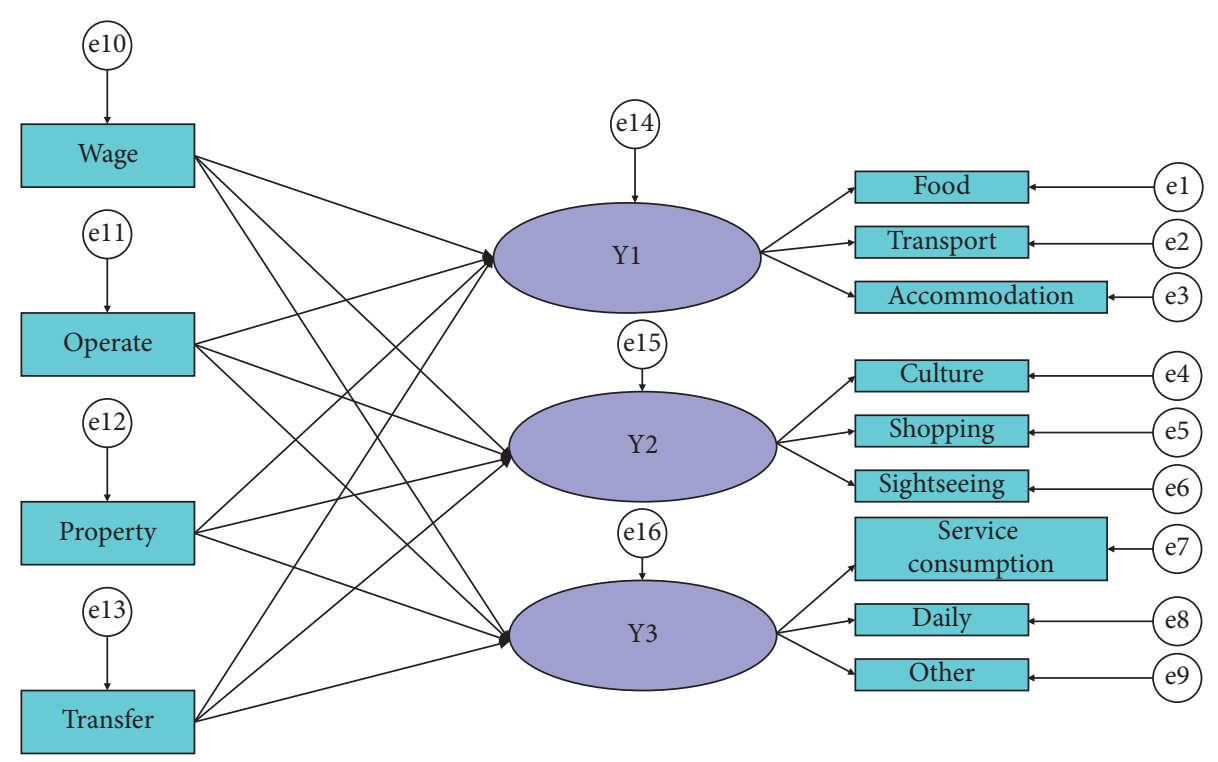

FIGURE 5: Structural equation path diagram.

can be seen from Figure 4 [23], the Engel coefficient for rural areas is consistently higher than that for urban areas. The coefficient for rural areas has always been higher than that for urban areas, and only twice did rural areas catch up with urban areas, in 1983 and 1989. In 1983 and 1989 [24], the rural areas slightly caught up with the towns. After 1989, the Engel coefficient between rural and urban areas significantly widened, indicating that China's special urban-rural dichotomy has had a significant impact on the rural economy. This indicates that China's special urban-rural dichotomy 
TABLE 4: Measurement model parameter estimation table.

\begin{tabular}{|c|c|c|c|c|c|}
\hline Latent variable & Observed variable & Estimate & S.E. & Est./S.E. & $P$-value \\
\hline \multirow{3}{*}{ Basic consumption $\left(\mathrm{Y}_{1}\right)$} & Food & 0.684 & 0.018 & 38.18938 .189 & $\leq 0.001$ \\
\hline & Transport & 0.551 & 0.016 & 34.830 & $\leq 0.001$ \\
\hline & Accommodation & 0.036 & 0.015 & 2.350 & 0.019 \\
\hline \multirow{3}{*}{ Recreational consumption $\left(\mathrm{Y}_{2}\right)$} & Culture & 0.816 & 0.014 & 56.414 & $\leq 0.001$ \\
\hline & Shopping & 0.200 & 0.011 & 17.487 & $\leq 0.001$ \\
\hline & Sightseeing & 0.091 & 0.012 & 7.729 & $\leq 0.001$ \\
\hline \multirow{3}{*}{ Other consumption $\left(\mathrm{Y}_{3}\right)$} & Services & 0.217 & 0.012 & 17.828 & $\leq 0.001$ \\
\hline & Daily & 0.835 & 0.013 & 64.507 & $\leq 0.001$ \\
\hline & Other & 0.119 & 0.011 & 10.813 & $\leq 0.001$ \\
\hline
\end{tabular}

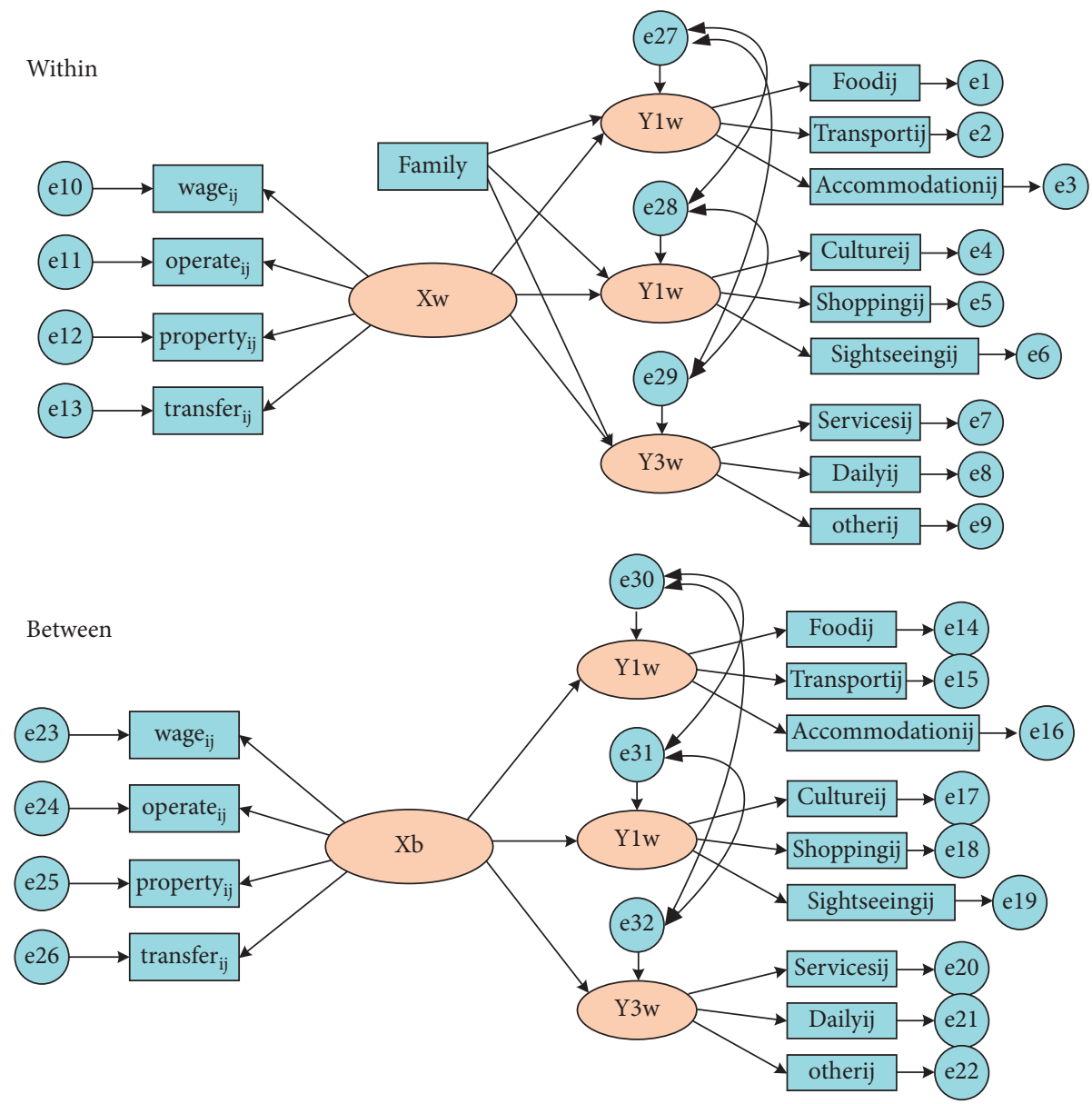

FIgURE 6: Hierarchical structural equation path diagram.

has had an impact on residents' consumption behavior. It was not until 2004 that the gap between urban and rural areas began to widen. It was only after 2004 that the gap between urban and rural areas began to narrow. From 2012 to the present, the difference between the urban and rural Engel coefficients has remained at around 3\% [25]. The gap between urban and rural areas has remained at around 3\%. Tables 2 and 3 show the income structure of urban residents and the income structure of rural residents, respectively.

There is a positive correlation between various types of income and various types of consumption. Compared with other types of income, the relationship between wage income and various types of consumption is closer, which can reflect that most of the income sources of my country's residents are wage income. Necessary consumption in daily life is closely related to all types of income. The correlation between property income and tourism, culture, and entertainment consumption is relatively strong. This may be because families with more family property income are wealthier than other families, so they spend more on enjoyment consumption such as tourism. It seems that transfer income has a relatively small correlation with many 
TABle 5: Parameter estimation table for the measurement model of household tourism consumption latent variables.

\begin{tabular}{|c|c|c|c|c|c|}
\hline Latent variable & Observed variable & Estimate & S.E. & Est./S.E. & $\mathrm{P}$-value \\
\hline \multicolumn{6}{|c|}{ Family level } \\
\hline \multirow{4}{*}{ Basic consumption $\left(\mathrm{YW}_{1}\right)$} & Food & 0.612 & 0.049 & 12.409 & $\leq 0.001$ \\
\hline & Transport & 0.583 & 0.043 & 13.590 & $\leq 0.001$ \\
\hline & Accommodation & 0.034 & 0.011 & 3.017 & 0.003 \\
\hline & Culture & 0.805 & 0.061 & 13.192 & $\leq 0.001$ \\
\hline \multirow[t]{2}{*}{ Recreational consumption $\left(\mathrm{YW}_{2}\right)$} & Shopping & 0.193 & 0.023 & 8.377 & $\leq 0.001$ \\
\hline & Sightseeing & 0.082 & 0.026 & 3.154 & 0.002 \\
\hline \multirow{3}{*}{ Other consumption $\left(\mathrm{YW}_{3}\right)$} & Services & 0.198 & 0.028 & 7.191 & $\leq 0.001$ \\
\hline & Daily & 0.819 & 0.056 & 14.576 & $\leq 0.001$ \\
\hline & Other & 0.110 & 0.022 & 4.897 & $\leq 0.001$ \\
\hline \multicolumn{6}{|c|}{ Regional level } \\
\hline \multirow{4}{*}{ Basic consumption $\left(\mathrm{YB}_{1}\right)$} & Food & 0.946 & 0.414 & 2.287 & 0.022 \\
\hline & Transport & 0.857 & 0.288 & 2.977 & 0.003 \\
\hline & Accommodation & -0.552 & 3.682 & 0.150 & 0.881 \\
\hline & Culture & 0.969 & 0.097 & 9.973 & $\leq 0.001$ \\
\hline \multirow[t]{2}{*}{ Recreational consumption $\left(\mathrm{YB}_{2}\right)$} & Shopping & 0.927 & 0.142 & 6.554 & $\leq 0.001$ \\
\hline & Sightseeing & 0.885 & 0.194 & 4.556 & 0.002 \\
\hline \multirow{3}{*}{ Other consumption $\left(\mathrm{YB}_{3}\right)$} & Services & 0.947 & 0.087 & 10.819 & $\leq 0.001$ \\
\hline & Daily & 0.990 & 0.223 & 4.445 & $\leq 0.001$ \\
\hline & Other & 0.932 & 0.047 & 19.875 & $\leq 0.001$ \\
\hline
\end{tabular}

consumptions. This may be because the households receiving transfer income are not very wealthy, so they spend slightly less on tourism and culture.

4.4. Structural Equation Modeling Analysis. Next, in order to provide a more realistic picture of the relationship between the structure of household income and the structure of household tourism consumption, the structural equation model was used to generate three consumption structure latent variables for various subcategories of consumption and to analyze in detail the relationship between the four types of income structure and the tourism consumption structure latent variables. The relationship between the variables is shown in Figure 5.

The calculation results of the measurement model are shown in Table 4. From the point of view of variable significance, each observed significant variable of consumption can well explain the latent variable of consumption structure, and they are all significant at the $1 \%$ significance level. From the perspective of variable path coefficients, the coefficients of all explicit variables of consumption are all positive.

4.5. Multilevel Structural Equation Modeling. On the basis of the above model, adding covariate family $y_{i} j$ family characteristics in the structure of the household-level model, they are urban and rural categories (urbanj), family size (sizej), education (eduj), and work experience (expj). The variable relationship diagram is shown in Figure 6.

It can be seen from Table 5 that the relationship between the observed variables and the latent variables of tourism consumption structure is basically the same as above, and the estimated value at the regional level is slightly higher than that at the individual level [26]. As shown in Table 6, each observed significant variable of income can explain the
TABLE 6: Income latent variable measurement model parameter estimation table.

\begin{tabular}{lcrccc}
\hline $\begin{array}{l}\text { Latent } \\
\text { variable }\end{array}$ & $\begin{array}{l}\text { Observed } \\
\text { variable }\end{array}$ & Estimate & S.E. & $\begin{array}{c}\text { Est./ } \\
\text { S.E. }\end{array}$ & $\begin{array}{c}P- \\
\text { value }\end{array}$ \\
\hline \multicolumn{5}{c}{ Family level } \\
& Wage & 0.615 & 0.037 & 16.447 & $\leq 0.001$ \\
Income(XW) & Operate & 0.610 & 0.018 & 34.192 & $\leq 0.001$ \\
& Property & 0.632 & 0.032 & 19.886 & 0.003 \\
& Transfer & 0.452 & 0.024 & 18.458 & \\
& \multicolumn{2}{c}{ Regional level } \\
& Wage & 0.948 & 0.140 & 6.779 & $\leq 0.001$ \\
Income $(\mathrm{XB})$ & Operate & 0.913 & 0.246 & 3.712 & $\leq 0.001$ \\
& Property & 0.772 & 0.119 & 6.466 & 0.003 \\
& Transfer & 0.953 & 0.062 & 15.460 & $\leq 0.001$ \\
\hline
\end{tabular}

latent variable of income structure well, and they are all significant at the significance level of $1 \%$. The coefficient of influence of each significant variable of income is positive [27]. The four types of income have a positive effect on income. The coefficients of the structure are basically the same.

\section{Conclusions}

Income structure is the main reason that affects family tourism consumption. Through canonical correlation and structural equation model analysis, it can be seen that there is a significant correlation between the household income structure and tourism consumption structure in China. Wage income has a significant impact on all types of tourism consumption structure, indicating that distribution according to work is the mainstay in China under the circumstances, and wage income is still the main source of income for households. Operating income mainly affects basic tourism consumption and developmental tourism consumption. Property income mainly affects enjoyment 
tourism consumption. Transfer income only has an impact on basic catering and transportation consumption.

In terms of household microfactors, urban/rural category, household size, and educational attainment, all have a significant positive effect on tourism consumption, while work experience has a negative effect. Urban households are more likely to spend on all types of tourism than rural households and have the greatest influence on the basic items of the first category of tourism consumption. The structure of consumption is closely related to the size of the household, with the larger the household size, the greater the ability to spend on tourism, and still the greatest impact on the basic categories of consumption. The higher the level of education of the household as a whole, the higher the spending power on all types of consumption and the largest coefficient of influence on the enjoyment type of tourism consumption.

The relationships between the latent variables of the consumption structure vary. The closest relationship between the consumption structure latent variables, both at the household level and at the regional level, in the structural relationship between tourism consumption is between enjoyment-based consumption and other consumption items. At the household level, higher incomes may lead households to increase both types of consumption. At the regional level, expenditure on other items in tourism activities may have some synergistic relationship with enjoyment-based consumption. In the future, we plan to carry out overly deep learning household travel consumption impact analysis to further improve the model analysis.

\section{Data Availability}

The datasets used during the current study are available from the corresponding author on reasonable request.

\section{Conflicts of Interest}

The authors declare that they have no conflict of interest.

\section{References}

[1] D. Wang and G. Sun, "Research on influencing factors of tourism consumption of urban families in China," Age, vol. 3, pp. 1-563, 2019.

[2] C. Kelly, "Beyond 'a trip to the seaside': exploring emotions and family tourism experiences," Tourism Geographies, pp. 1-22, 2020.

[3] B. Pikkemaat, M. Peters, and B. F. Bichler, "Innovation research in tourism: research streams and actions for the future," Journal of Hospitality and Tourism Management, vol. 41, pp. 184-196, 2019.

[4] Y. U. Ziping, "Health and wellness tourism in China: concept and development," Journal of Landscape Research, vol. 11, no. 6, 2019.

[5] M. Li, G. Lin, and X. Feng, "An interactive family tourism decision model," Journal of Travel Research, Article ID $00472875211056682,2021$.

[6] J. Pei, K. Zhong, J. Li, and J. Xu, "ECNN: evaluating a clusterneural network model for city innovation capability," Neural Computing \& Applications, pp. 1-13, 2021.
[7] M. Á Oviedo-García, M. R. González-Rodríguez, and M. Vega-Vázquez, "Does sun-and-sea all-inclusive tourism contribute to poverty alleviation and/or income inequality reduction? The case of the Dominican Republic," Journal of Travel Research, vol. 58, no. 6, pp. 995-1013, 2019.

[8] Y. Liu, Y. Li, and L. Li, "A panel data-based analysis of factors influencing market demand for Chinese outbound tourism," Asia Pacific Journal of Tourism Research, vol. 23, no. 7, pp. 667-676, 2018.

[9] S. A. Drakopoulos, "The marginalization of absolute and relative income hypotheses of consumption and the role of fiscal policy," The European Journal of the History of Economic Thought, vol. 28, pp. 1-20, 2021.

[10] M. Yiğit, "Testing the validity OF absolute income hypothesis through average propensity to consume forturkish economy," Theoretical and Applied Studies On Turkish Economy, vol. I, p. 105, 2020.

[11] K. J. White, "Financial stress and the relative income hypothesis among black college students," Contemporary Family Therapy, vol. 42, no. 1, pp. 25-32, 2020.

[12] J. Bricker, J. Krimmel, and R. Ramcharan, "Signaling status: the impact of relative income on household consumption and financial decisions," Management Science, vol. 67, no. 4, pp. 1993-2009, 2021.

[13] R. Holbrook and F. Stafford, "The propensity to consume separate types of income: a generalized permanent income hypothesis," Econometrica, vol. 39, no. 1, pp. 1-21, 1971.

[14] N. Wang, "Generalizing the permanent-income hypothesis: revisiting Friedman's conjecture on consumption," Journal of Monetary Economics, vol. 53, no. 4, pp. 737-752, 2006.

[15] P. Sunley, "What's behind the models? A review of The Spatial Economy," Journal of Economic Geography, vol. 1, no. 1, pp. 136-139, 2001.

[16] K. V. Murthy, "Arguing a case for Cobb-Douglas production function," Review of Commerce Studies, vol. 20, p. 21, 2002.

[17] W. T. Hung, J. K. Shang, and F. C. Wang, "A multilevel analysis on the determinants of household tourism expenditure," Current Issues in Tourism, vol. 16, no. 6, pp. 612-617, 2013.

[18] I. Burić and L. E. Kim, "Teacher self-efficacy, instructional quality, and student motivational beliefs: an analysis using multilevel structural equation modeling," Learning and Instruction, vol. 66, Article ID 101302, 2020.

[19] K. Otani, Y. Deng, P. A. Herrmann, and R. S. Kurz, "Patient satisfaction, quality attributes, and organizational characteristics: a hierarchical linear model approach," Journal of $\mathrm{Pa}$ tient Experience, vol. 7, no. 5, pp. 801-806, 2020.

[20] N. K. Bowen and S. Guo, Structural Equation modeling, Oxford University Press, Oxford, UK, 2011.

[21] H. Bin and X. Lei, "Housing Comparison and Household Consumption," Statistical Research, vol. 32, no. 12, 2015.

[22] X. Liu, Y. Li, and F. Feng, "Thirty years of legal theory in China's reform and opening up: 1978-2008," Law and Social Development, vol. 5, no. 3, 2008.

[23] Q. Ding, W. Cai, C. Wang, and M. Sanwal, "The relationships between household consumption activities and energy consumption in China- an input-output analysis from the lifestyle perspective," Applied Energy, vol. 207, pp. 520-532, 2017.

[24] S. R. Baker, R. A. Farrokhnia, S. Meyer, M. Pagel, and C. Yannelis, "How does household spending respond to an epidemic? Consumption during the 2020 COVID-19 pandemic," The Review of Asset Pricing Studies, vol. 10, no. 4, pp. 834-862, 2020. 
[25] L. V. Dutra, D. d. C. Morais, R. H. S. Santos, S. d. C. C. Franceschini, and S. E. Priore, "Contribution of the production for self-consumption to food availability and food security in households of the rural area of a Brazilian city," Ecology of Food and Nutrition, vol. 57, no. 4, pp. 282-300, 2018.

[26] A. D. Grotzinger, M. Rhemtulla, R. de Vlaming et al., "Genomic structural equation modelling provides insights into the multivariate genetic architecture of complex traits," $\mathrm{Na}$ ture human behaviour, vol. 3, no. 5, pp. 513-525, 2019.

[27] Z. I. Santini, P. E. Jose, E. York Cornwell et al., "Social disconnectedness, perceived isolation, and symptoms of depression and anxiety among older Americans (NSHAP): a longitudinal mediation analysis," The Lancet Public Health, vol. 5, no. 1, pp. e62-e70, 2020. 